\title{
INDOOR MONITORING APPLICATIONS USING FIXED AND MOBILE WIRELESS SENSORS
}

\author{
Mircea lonel Strutu, Dan Popescu \\ Politehnica University of Bucharest, Splaiul Independentei 313, Sector 4, Bucharest, Romania 060042, \\ strutu_mircea@yahoo.com,dan_popescu_2002@yahoo.com,www.pub.ro
}

\begin{abstract}
This paper proposes the idea of a wireless sensor network with applicability in monitoring systems. The goal of the project is to build a monitoring system capable of data gathering which can benefit from both characteristics of fixed and mobile nodes. To become flexible, the mobile node has a modular structure with four levels. The flexibility offered by the mobile nodes increases the performance of the entire data acquisition system. These act as autonomous devices that cooperatively monitor physical or environmental conditions. In order to implement the data acquisition and communication functions, the solution we agreed on has been to use the MTS400 sensor board. Mobile nodes communicate to a central node individual data and statistical indicators over a period. The proposed energy-aware architecture was tested and validated in an indoor experiment.
\end{abstract}

Keywords: monitoring system, wireless sensor network, multi-hop mesh network, mobile node, reliability, redundancy, gateway.

\section{INTRODUCTION ${ }^{1}$}

A wireless sensor network is typically composed of small, matchbox-sized devices, known as "motes" which support a range of environmental sensing capabilities and can be randomly and densely deployed. Sensor capabilities may include temperature, light, humidity, radiation, the presence of biological organisms, geological features, seismic vibrations and more. Recent development made possible to make these components small, powerful and energy efficient and they can now be manufactured cost-effectively and in large quantities for a wide range of specialized telecommunications applications. Very small in size, the sensor nodes are capable of gathering, processing, and communicating data to other nodes and to the outside world. Based on the information handling capabilities and compact size of the sensor nodes, sensor networks are often referred to as "smart dust" [1].

Benefiting from a large variety of sensors, sensor networks have many fields of applications, including health, agriculture, geology, military, home and emergency management. The limited battery life of a sensor node raises the efficient energy

\footnotetext{
${ }^{1}$ This work was supported in part by DocInvest Project ID 76813/ POSDRU_Romania (Burse doctorale: investitii in cercetare-inovaredezvoltare pentru viitor)
}

consumption as a key issue in wireless sensor networks [2]. Thus, energy efficiency is a primary requirement in a wireless sensor network and a major design parameter in medium access control protocols for WSN [3].

Wireless sensor networks have attracted a wide interest from industry due to their diversity of applications. Sensor networks are pervasive by nature; the number of nodes in a network is nearly boundless. As a consequence, it is becoming increasing difficult to discuss typical requirements regarding hardware issues and software support. This is particularly problematic in a multidisciplinary research area such as wireless sensor networks, where close collaboration between users, application domain experts, hardware designers, and software developers is needed to implement efficient systems [2]. Therefore, a key to realise this potential is multi-hop mesh networking, which enables scalability and reliability.

One of the most frequently design aims is to minimizing the power consumption in different functional layers of wireless nodes. Many studies have been proposed techniques for energy efficient data processing, communication and storage. As a result, a dynamic balancing between these functions is necessary [4], [5], [6].

The variety of sensing capabilities offered by these devices also provides an opportunity to gain an unprecedented level of information about a target 
area, be it a room, building or outdoor. Wireless sensor networks (WSN) are fundamentally a tool to measure the spatial and temporal characteristics of any phenomena [7].

Because of the presence of people, for particular case of indoor applications, low energy of radiations also is an important requirement. High RF power levels, that imply high energy-consumption, are undesirable for interference with other networks and for health hazards of people due to continuous exposure to radiation antenna radiation levels of the wireless equipment) especially in AAL (homecare monitoring).

Therefore the communication range of BSN is very small (up to $8 \mathrm{~m}$ ). Now, thanks to the ITC development, intelligent systems are used to support elderly and chronically ill in all aspects of daily life, at home. For example, European program "Ambient Assisted Living Joint Programme" (AAL), in progress, aims to extend the time in which the elderly can live independently in their home by assisting them in carrying out activities of daily living [8]. This means that the environment will be characterized by embedded technology, sensitive, adaptive and responsive to the target people.

In the near future it can be expected that buildings will be equipped with a range of wireless sensors functioning as a part of an overall building management system. It expects such information could be used for a variety of purposes, including guiding occupants to the nearest safe exit in case of emergency [9].

\section{MONITORING SYSTEMS}

The purpose of deploying a WSN is to collect relevant data for processing and reporting. In this paper are presented the incipient stages of a more complex project we started to develop. Trying to apply a mobile monitoring system to a multi-storey building offers great advantages, but it also raises some questions.

One of the primary advantages of deploying a wireless sensor network is its low deployment cost and freedom from requiring a messy wired communication backbone, which is often infeasible or economically inconvenient [10]. Cattivelli et al [11] study the problem of distributed estimation in order to evaluate some parameter of interest from measurements in an adaptive network. For the global solution they propose a distributed diffusion algorithm based on recursive least-squares (obtain estimates that are close to the global solution).

On the other hand, one of the first questions to be answered is how to deal with obstacles and how to achieve communication over long distances. Energy consumption or, in general, resource management is of critical importance to these networks. Each node will collect raw data from the environment, and communicate with each other to perform a task. Each node has a sensing radius within which it can gather data and a communication radius within which it can communicate with each other. Trying to reach a remote location to gather valuable data can be done using a mobile platform like the one we have (Fig. 1).

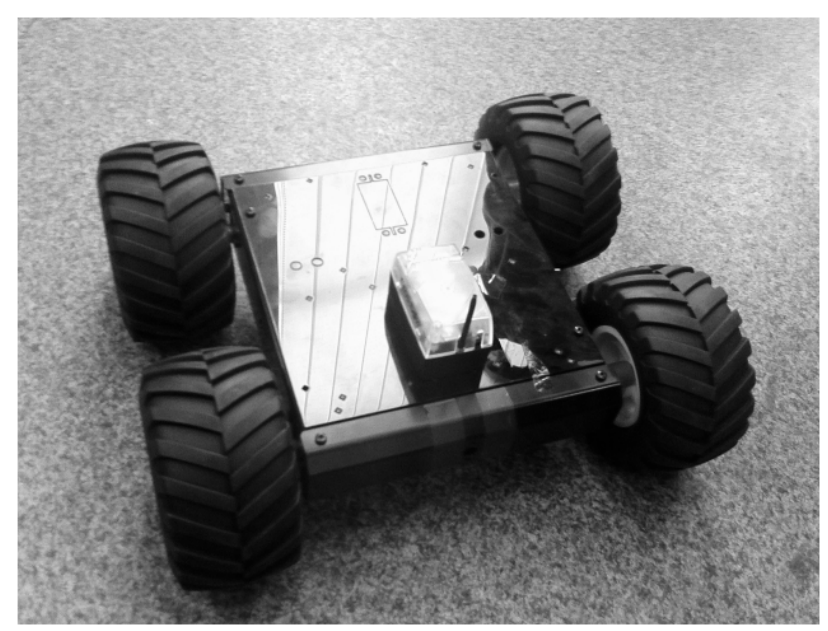

Fig. 1 - Mobile platform equipped with wireless sensor

Using many mobile nodes to achieve a very good coverage of the monitored space can be a good solution but it can become costly in terms of energy consumption and maintenance. One important criterion for being able to deploy an efficient sensor network is to find optimal node placement strategies. Cost driven development resulted in choosing a more versatile topology for our network consisting in both fixed and mobile nodes. This approach of the problem gives us the possibility to easily adapt the network for the requirements of a monitoring system, but also to make it act as an alerting system against a predefined alert situation. An alert situation can be defined as anything from a fire to an earthquake.

The mobile node architecture is a modular one and contains four functional levels: communication, processing \& management, data acquisition and power supply \& traction (Fig. 2).

Some of the mobile nodes characteristics may prove to be very important for our monitoring solution. The main characteristic is that the topology is not fixed. During functioning, nodes will change position or go offline.

The notion of area coverage can be considered as a measure of quality of service (QoS) in a sensor network, for it means how well each point in the sensing field is covered by the sensing ranges. Our solution presents a practical approach to the 
coverage problem giving the opportunity for the mobile nodes to demonstrate their great capability in monitoring large areas.

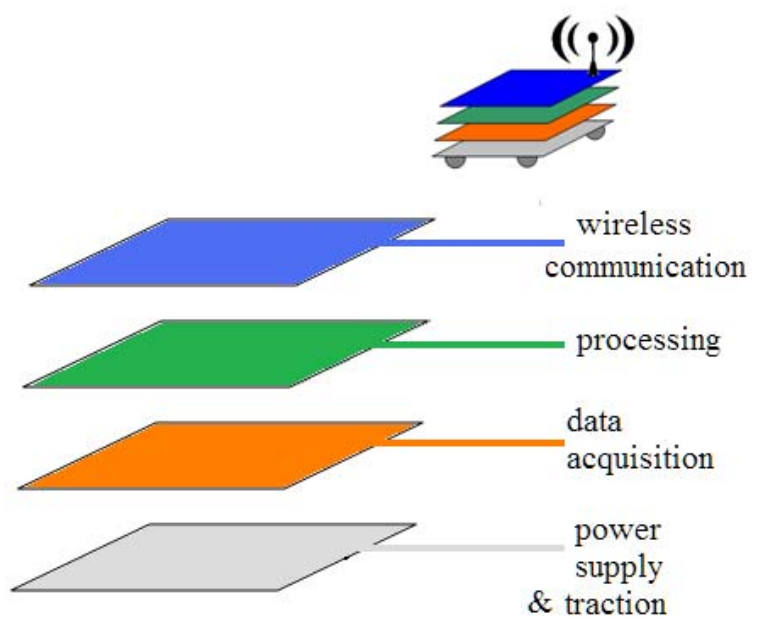

Fig. 2 - Mobile node architecture

Because mobile sensors give us a greater degree of freedom, they can also be quickly deployed and can easily cover a certain area even when human presence may be impossible. Our desired network consists mainly in fixed nodes and also few mobile nodes in order to perform specific tasks. All the nodes will be connected to the gateway and through this to a processing centre which can be accessed using a user interface (Fig. 3).

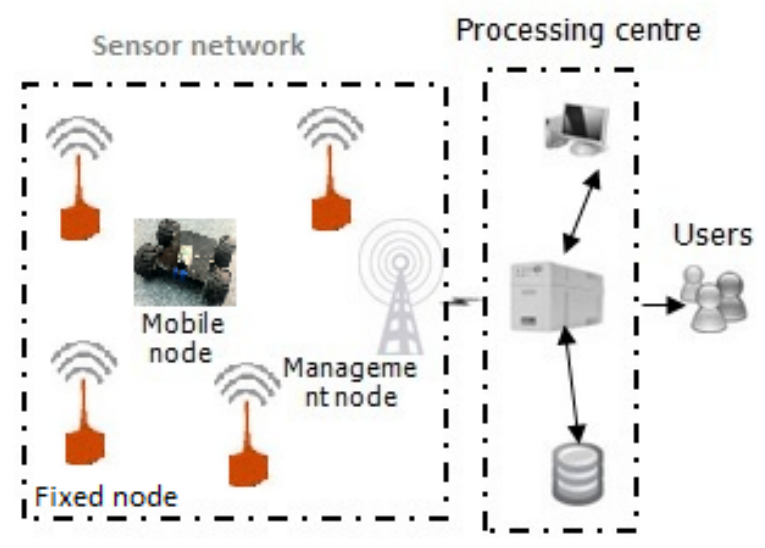

Fig. 3 - Mobile sensor alarm system deployment

The ultimate purpose of implementing a monitoring system is developing a good alerting system capable of early detection of potentially dangerous situations. The end users of the monitoring and alerting system should be the people living and working in the monitored area, in our case, inside the monitored building.

Using a mixture of fixed and mobile nodes induces a great deal of redundancy which makes the entire wireless network more reliable and provides the monitoring system which the means to react in case of emergency. The mobile nodes can also act as an on the scene monitoring system in case a potentially dangerous situation is detected. Having a mobile platform equipped with sensors capable of reaching the place where the problem occurred in case of emergency can be of great help for correctly evaluating the situation and tacking the proper actions against it as soon as possible.

A sensible matter is dealing with false alerts. The alerting system should be capable of dissociating between real alert situations and sensor associated errors. False alarms may result in causing unnecessary panic and bringing resources (such as emergency services) when they are not needed. These kind of situations should be avoided in order to keep a good level of confidence for the implemented alerting solution.

The best way to avoid unwanted triggers is to go through a vast number of test cases and to use a fault detection mechaninsm to validate data obtained from the sensors.

Other constarints for the chosen solution will be the motes size and weighth, the battery life, it should be long enough to perform the tasks without human intervention and also the battery life of the mobile platform.

\section{CHOSEN SOLUTION}

Our goal is to build a monitoring system which can benefit from both characteristics of fixed and mobile nodes. So, our decision must be made having in mind the fact that the motes should be capable to use both as fixed and mobile nodes without further preparation.

A typical wireless sensor network consists in spatially distributed sensors. These act as autonomous devices that cooperatively monitor physical or environmental conditions. The great advantage of using a wireless network is that you can forget about the messy wired communication which in most cases can be impractical and more costly.

Having in plan to use our sensor network as an environmental monitoring system, the first step in designing our solution has been to choose the needed sensors. In order to achieve a good monitoring level for the environmental conditions indoor or outdoor, we needed to have our nodes equipped for gathering the following parameters: temperature, light, humidity, barometric pressure and also seismic.

To conduct our experiments, we decided to use in the early stages only four motes, three of them as fixed nodes and another one a mobile node on our remotely operated platform [12].

In order to choose the proper technology for our 
communication infrastructure, a comparison between available wireless communication technologies was made (Fig. 4) [13].

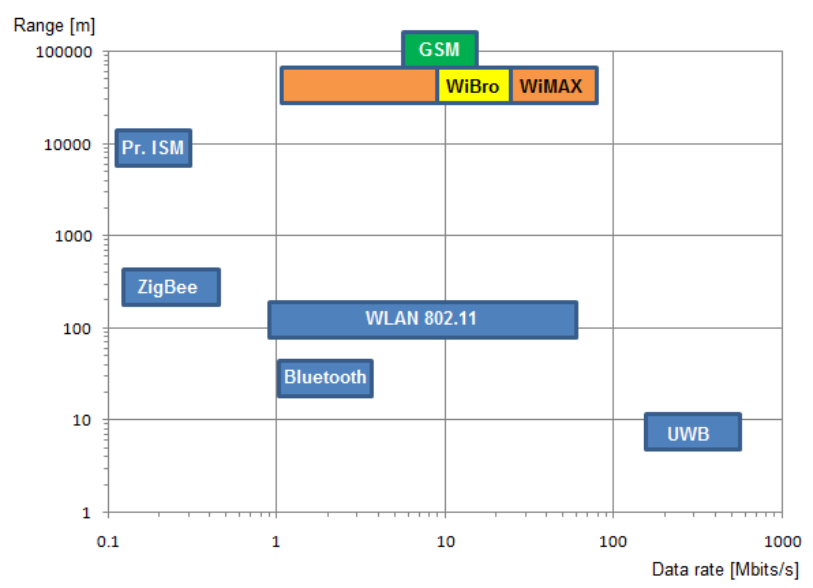

Fig. 4 - Graphical representation of wireless technologies segmentation

Based on requires demanded by the mobile sensor network, this representation can be seen like a guide from which, can be chosen the suitable wireless technology for a communication module.

For technology selection, two important features are represented on the axes, namely distance and data rate, both in logarithmic scale. The area of each technology representation figures the level of energy consumption approach in design of the respective technology.

A small area represents a standard with energy consumption optimization; instead a wide area represents a technology which wasn't designed to be energy efficient.

An example of choice of the proper technology for communication function is presented in [13] where the eliminatory demand was low power consumption. As one can see in Table 1, for low data rate and low range, we remain only with the option for communication infrastructure technology ZigBee, because, practically, it was especially develop for such application as mobile sensor networks.

Table 1. Low power wireless technologies

\begin{tabular}{|c|c|l|c|}
\hline \multicolumn{2}{|c|}{} & \multicolumn{2}{|c|}{ Range } \\
\cline { 3 - 4 } \multicolumn{2}{|c|}{} & Low & Wide \\
\hline \multirow{2}{*}{} & Low & ZigBee & GSM(GPRS) \\
\cline { 2 - 4 } & High & UWB & $\begin{array}{c}\text { WiBro, } \\
\text { GSM(HSDPA) }\end{array}$ \\
\hline
\end{tabular}

If the sink is far from the event, the co-operative communication is one of the methods to ensure communication task in energy-aware applications [14], [15].
In order to minimize the total energy consumption a multihop routing is possible. That implies the cooperation of some nodes to communicate data from a node away to the gateway. So, if a mobile node is outside of range of communication or an obstacle is interposed between the node and the gateway, another node can act as a relay to gateway to ensure communication.

The solution we agreed on has been to use the MTS400 sensor board (Fig. 5).

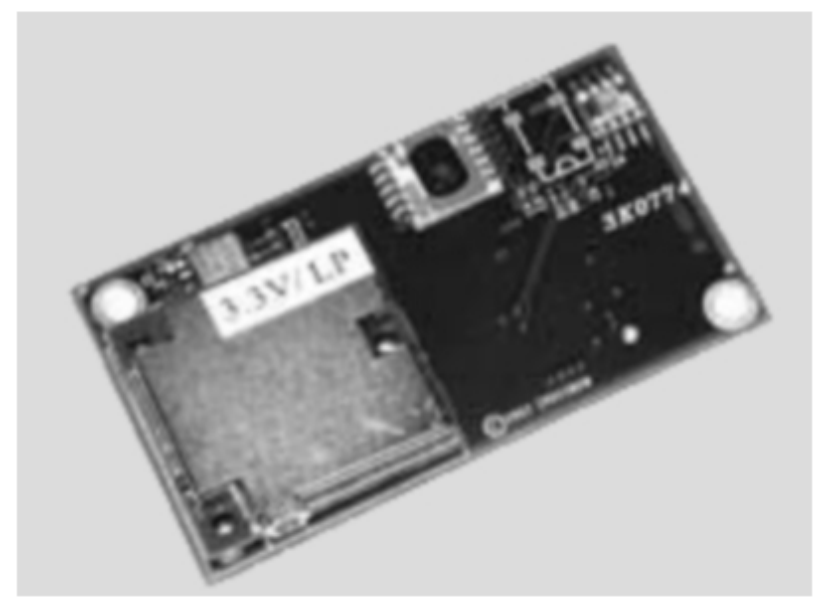

Fig. 5 - MTS400 Sensor Board

The MTS400 environmental sensor board offers five basic environmental sensing parameters and an optional GPS module (only available for MTS420). One of the main characteristics is that they are energy-efficient devices so that they can provide extended battery-life and performance wherever low maintenance field-deployed sensor nodes are required. These versatile sensor boards are intended for a wide variety of applications ranging from a simple wireless weather station to a full mesh network of environmental monitoring nodes. Applicable industries include agricultural monitoring, art preservation, environmental monitoring, sensor location mapping (available for GPS equipped MTS420).

According to the MTS420/400 Datasheet [16], some of the sensor board characteristics are as follows:

Dual-axis accelerometer: acceleration range; resolution: $\pm 2 \mathrm{~g}$; $2 \mathrm{mg}$ at $60 \mathrm{~Hz}$; sensitivity $167 \mathrm{mV} / \mathrm{g}$, $\pm 17 \%$,

Barometric pressure sensor: pressure range; resolution: 300-1100 mbar; 0.01 mbar; accuracy: $\pm 1.5 \%$ at $25^{\circ} \mathrm{C}$

Ambient light sensor: TAOS TSL2550D; spectral response: $400-1000 \mathrm{~nm}$, similar to human eye.

Relative humidity and temperature sensor: Sensirion SHT11; humidity range; resolution: 0100\% RH; $0.03 \%$ RH; absolute $\mathrm{RH}$ accuracy: \pm $3.5 \% \mathrm{RH}$; temperature accuracy: $\pm 0.5^{\circ} \mathrm{C}$ at $25^{\circ} \mathrm{C}$. 
The average operating range for the described sensors is between 2.5 and 3.5 volts [17].

The sensor node (MicaZ), manufactured by Crossbow, has a $7.3 \mathrm{MHz}$ Atmega128L processor, $128 \mathrm{~Kb}$ of code memory, $4 \mathrm{~Kb}$ of data memory, and a Chipcon CC2420 radio which supports the 802.15.4/ZigBee WPAN protocol (transmits up to 250 kilobits per second and an outdoor transmission range of approximately $30 \mathrm{~m}$ ) [17]. The dimensions of the node are $58 \mathrm{~mm} \times 32 \mathrm{~mm}$ and have reduced weight, making them ideal for mobile platforms (Fig. 6).

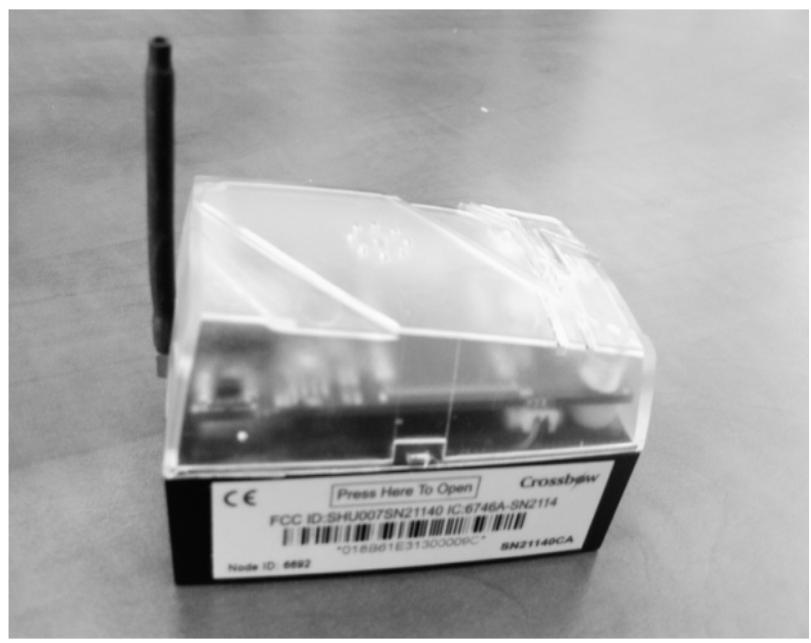

Fig. 6 - Wireless network node equipped with MTS400 sensor

The sink node is connected to a computer using a USB cable and remains permanently connected in order to transmit data to the database. The gateway's role is to aggregate the data from the network, interface the host, LAN, or the Internet, and act as a portal to monitor performance and configurable network parameters [18].

\section{EXPERIMENTAL DATA}

All the experiments have been made indoor using our faculty building as a test ground for the sensor network. The first stage of the experiment consisted in placing the sensor nodes inside the building, so that they can communicate with each other and to the gateway. Once the fixed nodes have been deployed, we started monitoring the data acquisition using MOTE-VIEW. This is an interface, client layer, between a user and a deployed network of wireless sensors. Once they are turned on, the sensor nodes appear in a list of available nodes on our interface. They can be identified by their unique id.

The first task is to set the update rate for each node. For the first experiment we used a data rate of 10 seconds for all our nodes. Every node will automatically appear in the upper left corner of the MOTE-VIEW interface. Each of them is assigned an ID and a Name for easy identifying within the network. The ID can be also seen on the side of each node (Fig. 6). Once the preparations have been made, real time data from each node are available (Fig. 7).

For the second part of the experiment, we use our mobile platform equipped with one of the nodes to gather data while moving inside the building. The mobile node $(\mathrm{Id}=6692)$ has been sending data once every 10 seconds to the gateway directly, or via one of the other nodes.

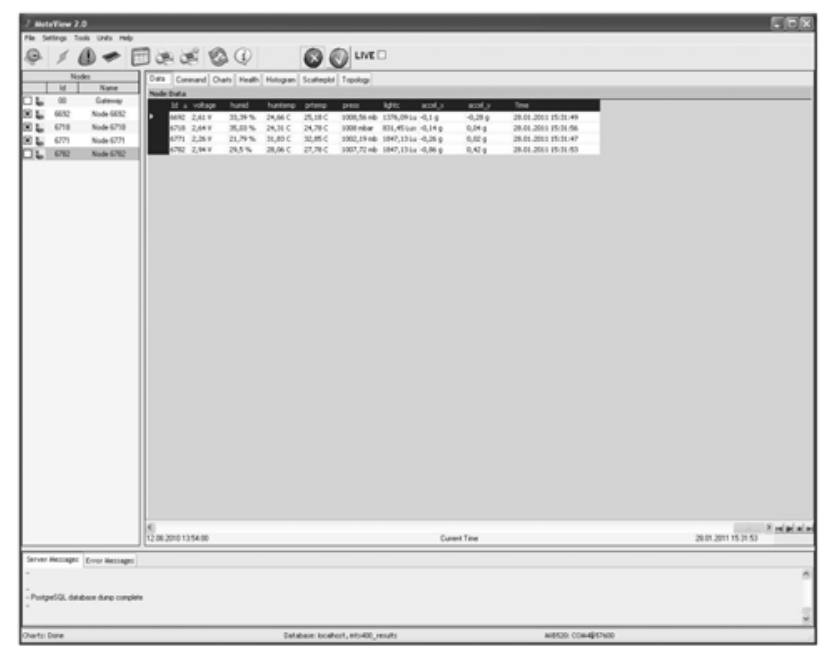

Fig. 7 - Data acquisition using MOTE-VIEW

In Table 2, the following parameters can be tracked over an interval of 5 minutes, 15 seconds: temperature $[\mathrm{C}]$, pressure [mbar], humidity [\%]. During this period of time, the platform has been programmed to move inside the building.

Some data has been lost because of the poor reception over long distances and thick walls. The advantage of having the possibility to send data to the gateway through another node is underlined by the fact that many data have been gathered this way (as it is shown by the parent column in Table 2).

From the harvested data, we can also automatically generate graphs of a sensor reading against time for one node or a set of nodes. For our experiment, we generated some charts to determine the data change in time for our mobile and fixed nodes. For example, we have generated the temperature evolution graph for the mobile node (Fig.8), and for all the nodes, comparatively (Fig. 9). Similarly, pressure and humidity evolutions are presented in Fig. 10 and Fig. 11, respectively.

As a result, some major temperature changes can be seen over the period of time (14:52:35 14:57:50). This is the interval when the mobile platform moved inside the building gathering data. Similar graphs have been generated for the other monitored parameters such as: light, humidity and pressure. 
Table 2. Mobile wireless sensor data

\begin{tabular}{|c|c|c|c|c|c|}
\hline Id & Time & Parent & $\begin{array}{c}\text { Humidity } \\
{[\%]}\end{array}$ & $\begin{array}{c}\text { Temp } \\
\text { [C] }\end{array}$ & $\begin{array}{c}\text { Press } \\
\text { [mbar] }\end{array}$ \\
\hline $669214: 52: 35$ & 0 & 39,1 & 25,3 & 1008,3 \\
\hline $669214: 52: 45$ & 0 & 40,5 & 25,33 & 1008,2 \\
\hline $669214: 52: 55$ & 0 & 34,9 & 25,25 & 1008,3 \\
\hline $669214: 53: 05$ & 0 & 34,1 & 25,24 & 1008,2 \\
\hline $669214: 54: 13$ & 6782 & 27,4 & 25,03 & 1008,3 \\
\hline $669214: 55: 02$ & 0 & 25,5 & 24,49 & 1008,3 \\
\hline $669214: 55: 11$ & 0 & 26,7 & 24,48 & 1008,4 \\
\hline $669214: 55: 31$ & 6718 & 28,2 & 24,4 & 1008,3 \\
\hline $669214: 55: 41$ & 6718 & 28,9 & 24,34 & 1008,5 \\
\hline $669214: 56: 00$ & 0 & 29,4 & 24,25 & 1008,5 \\
\hline $669214: 56: 21$ & 0 & 29,6 & 24,31 & 1008,6 \\
\hline $669214: 56: 31$ & 0 & 29,5 & 24,34 & 1008,5 \\
\hline $669214: 56: 50$ & 0 & 29,7 & 24,41 & 1008,3 \\
\hline $669214: 57: 02$ & 6718 & 29,6 & 24,4 & 1008,4 \\
\hline $669214: 57: 23$ & 0 & 29,8 & 24,44 & 1008,3 \\
\hline $669214: 57: 32$ & 0 & 29,8 & 24,47 & 1008,2 \\
\hline $669214: 57: 50$ & 0 & 29,9 & 24,48 & 1008,2 \\
\hline
\end{tabular}

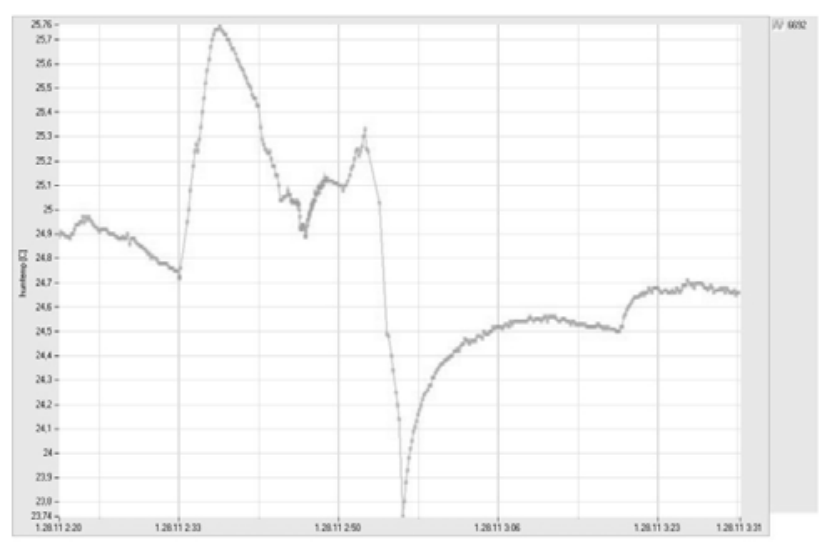

Fig. 8 - Temperature evolution measured by the mobile node

Because the rapid variations of measured values of parameters in the same location, for the evaluation of the real values some statistical indicators for humidity $(\mathrm{H})$, temperature $(\mathrm{T})$ and Pressure (P) are necessary. Thus, average values $\left(\mu_{H}\right.$, $\left.\mu_{T}, \mu_{P}\right)$ and estimations of standard deviations $\left(\sigma_{H}\right.$, $\sigma_{T}, \sigma_{P}$ ) are also provided by sensor node (Table 3 ):

$$
\mu=\frac{1}{n} \sum_{i=1}^{n} V_{i}, \quad \sigma=\sqrt{\frac{1}{n-1} \sum_{i=1}^{n}\left(V_{i}-\mu\right)^{2}}
$$

where $V_{\mathrm{i}}$ is an individual value of the measurement and $n$ is the number of these values.

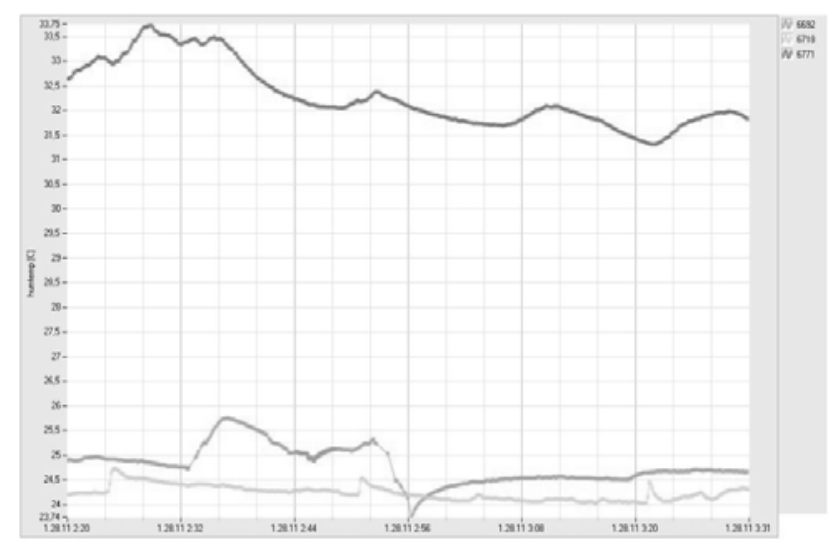

Fig. 9 - Temperature evolution over a period of time measured by 3 sensor nodes ( 2 fixed nodes and one mobile node)

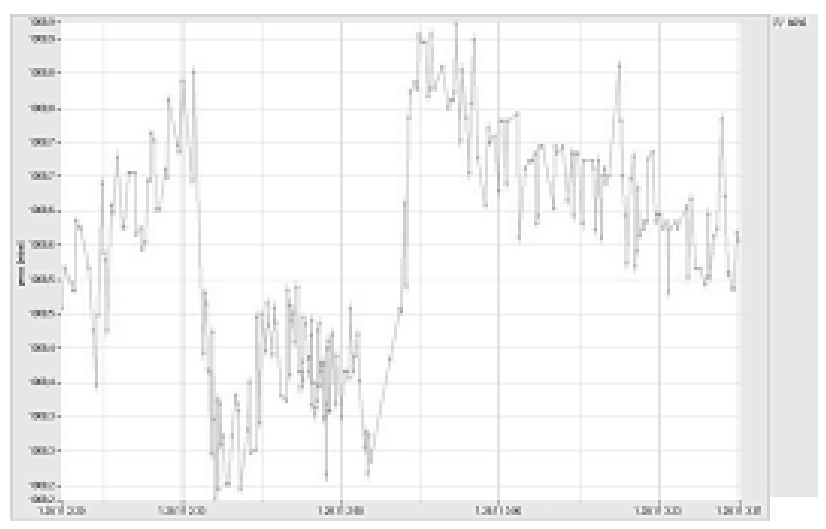

Fig. 10 - Pressure evolution measured by the mobile node

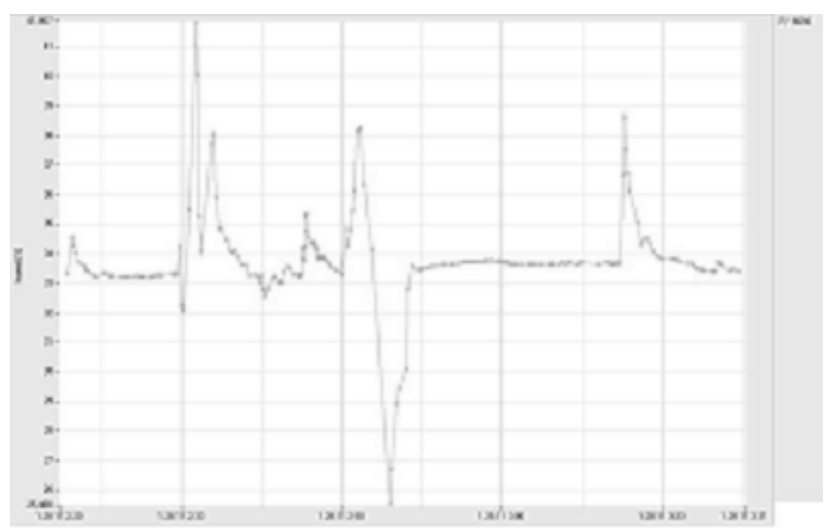

Fig. 11 - Humidity evolution (mobile node)

Table 3. Statistical indicators provided by mobile wireless sensor node

\begin{tabular}{|l|l|l|c|c|c|}
\hline $\begin{array}{l}\text { Stat. } \\
\text { Indic. }\end{array}$ & Period & Id & $\begin{array}{c}\text { Hum. } \\
{[\%]}\end{array}$ & $\begin{array}{c}\text { Temp. } \\
\text { [C] }\end{array}$ & $\begin{array}{c}\text { Press. } \\
\text { [mbar] }\end{array}$ \\
\hline$\mu$ & $14: 52: 35$ & 6692 & 29,7 & 24,4 & 1008,4 \\
& $14: 57: 50$ & & & & \\
\hline$\sigma$ & $14: 52: 35$ & 6692 & 4,23 & 0,46 & 0,12 \\
& $14: 57: 50$ & & & & \\
\hline
\end{tabular}

The topology of the mote network can be defined 
placing the nodes in the specific location on the map. This allows the user to visualize temperature or other data gathered by the sensor in an intuitive way using a wide range of colours for the representation of the parameter levels (Fig. 12).

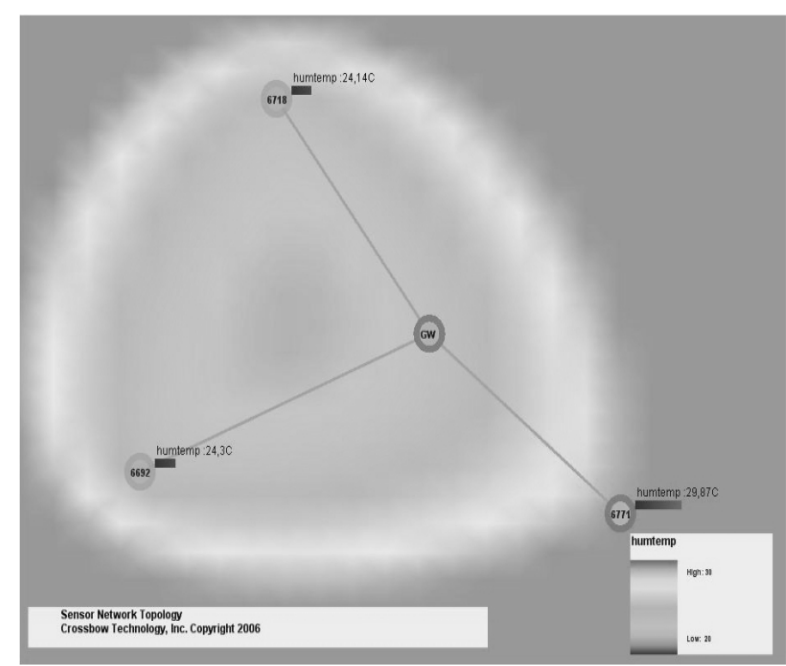

Fig. 12 - Temperature evolution measured by three nodes

In an outdoor implementation, using the GPS capability, the position of the nodes relatively to the gateway would be automatically available.

In Fig. 13 we can see a typical XMesh network diagram. We can see clearly that it has a great deal of redundancy. The real life situation looks more like the one in Fig. 14. So, we have to deal with the bottlenecks and avoiding obstacles in order to reassure that the sensorial coverage and communication coverage are satisfactory.

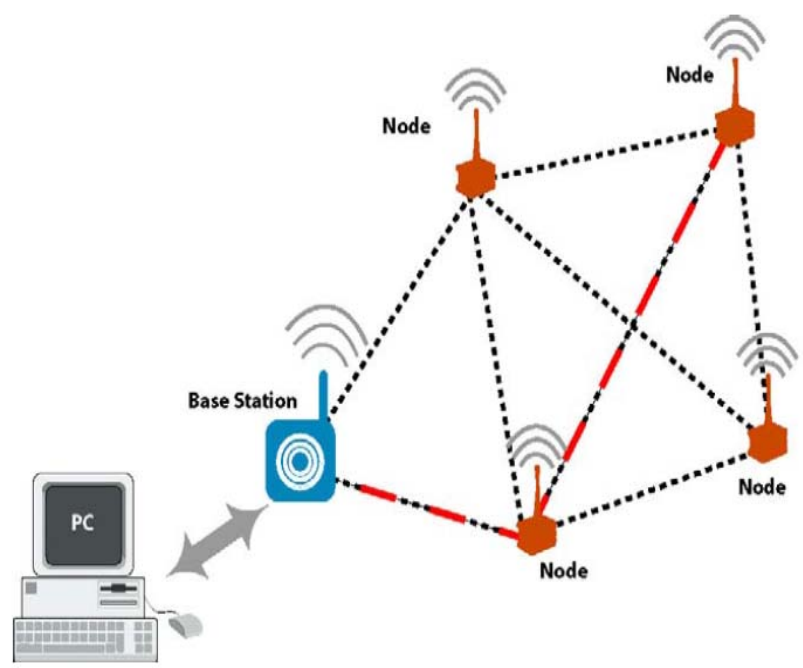

Fig. 13 - XMesh network diagram

The capability of our nodes to communicate to each other helped obtaining satisfactory communication coverage even when obstacles made direct communication to the gateway impossible.

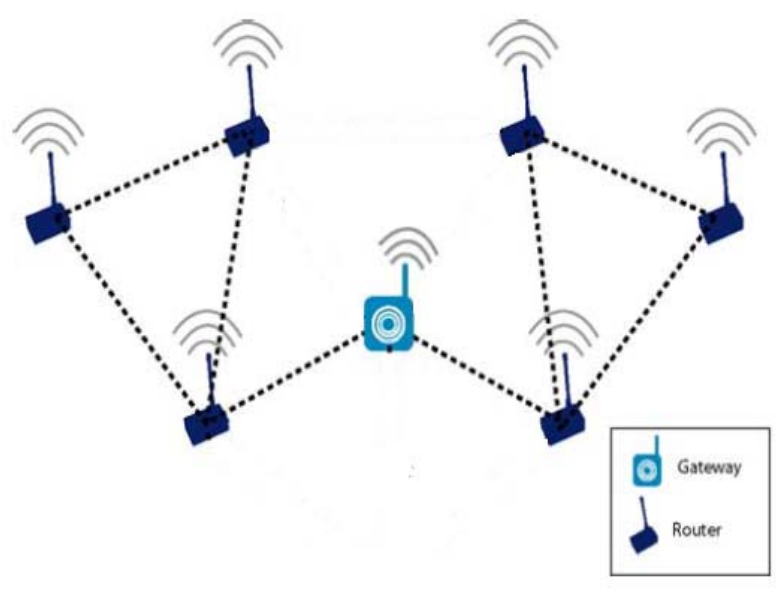

Fig. 14 - Diagram showing an indoor mesh topology with bottlenecks

Confronting our wireless sensor network with a series of test scenarios, the result was a tree shaped topology which could send data over long distances avoiding the obstacles. The mobile node proved to be a great enhancement giving us the possibility to send data over longer distances and replace the node in order to retrieve data from various places inside the building. Another possibility in case a fixed node goes offline is that an operator will place the mobile node in the same position as the fixed malfunctioning node to resume sending data from the same spot inside the building.

Dealing with false alerts is another issue. We can't let our monitoring system feed corrupt data to the alerting system. The false alerts should be avoided in order not to generate panic and distrust in the entire alerting system. The same principle as in case of replacing a malfunctioning node will be used.

In order to validate the alerting data from the fixed node, an operator will move the mobile node near the evaluated node's position. After a short while, the mobile node should start transmitting data similar to the fixed node. In case the alerting data are confirmed, the alerting system will turn the alarm on. In some cases, moving a mobile node near the fixed node's position will only prove that the data is corrupt and the fixed node needs maintenance.

In the third phase of our experiment, we want to test and to find a solution to a real life scenario when one of the retransmitting nodes stops functioning and a part of the network gets cut off from the gateway. A wireless network working inside a building may have many bottlenecks as it can be seen in Fig. 15. These can become a real problem if one of the retransmitting nodes stops working because, even if a part of the network is still operational, data cannot reach the gateway. 


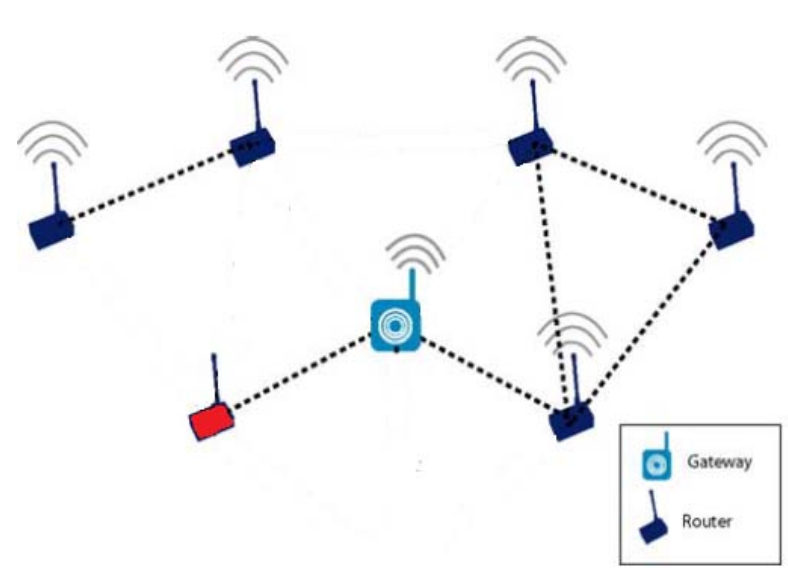

Fig. 15 - Diagram showing an indoor mesh topology with bottlenecks and a faulty node resulting in the loss of an entire branch of the network

In order to limit the risks of losing an entire branch of our network in case of a single malfunctioning node stops acting as an active router, we tried using our mobile node. The solution proved itself helpful (Fig. 16).

The transmission from the lost branch is resumed after placing the mobile node in the vicinity of the malfunctioning fixed node.

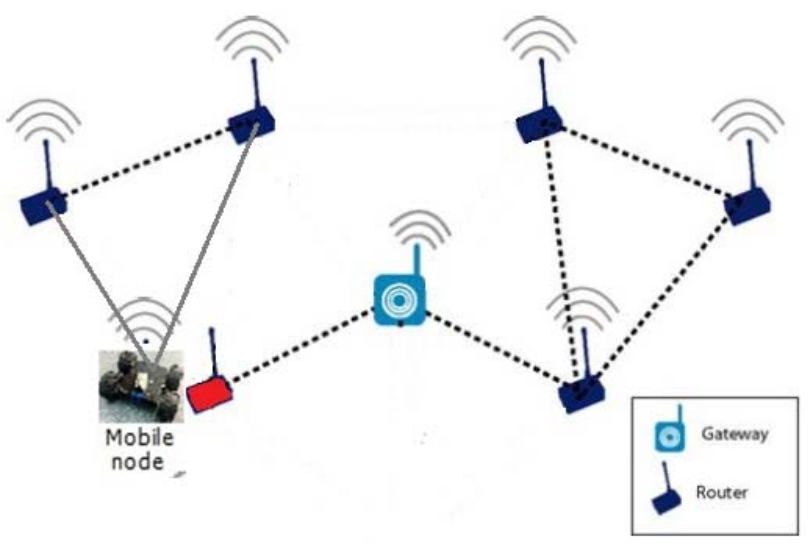

Fig. 16 - After the intervention of the mobile node, the transmission from the lost branch is resumed

So, in order to avoid losing data, we want to use our mobile nodes as retransmitting nodes. In case of a fixed node failure, the mobile node receives a command to automatically position itself so that the data transmission towards gateway may be resumed. The mechanism tolerates failures of random individual nodes in the network.

\section{FAULT DETECTION}

The goal of fault detection is to identify when a fault has occurred, to pinpoint the type of fault and its location.

The most simple and frequently used method for fault detection is the limit checking of a directly measured variable [19]. In this case, after rigorous testing, we established that the most frequent cause for a node malfunction is power failure. Some power sources can last more than others, so, when one of them runs off or is lower than the optimum working level of the sensors or the antenna, the node starts sending corrupt data and, ultimately, stops working, leaving the rest of the network with no communication link to the gateway. In order to prevent and predict this kind of failures, we implemented a limit checking fault detection system that triggers an alarm each time one of the nodes is dangerously close to the equipment functioning limits (Fig 17). As we said before, the operating range, for the described sensors, is between 2.5 and 3.5 volts.

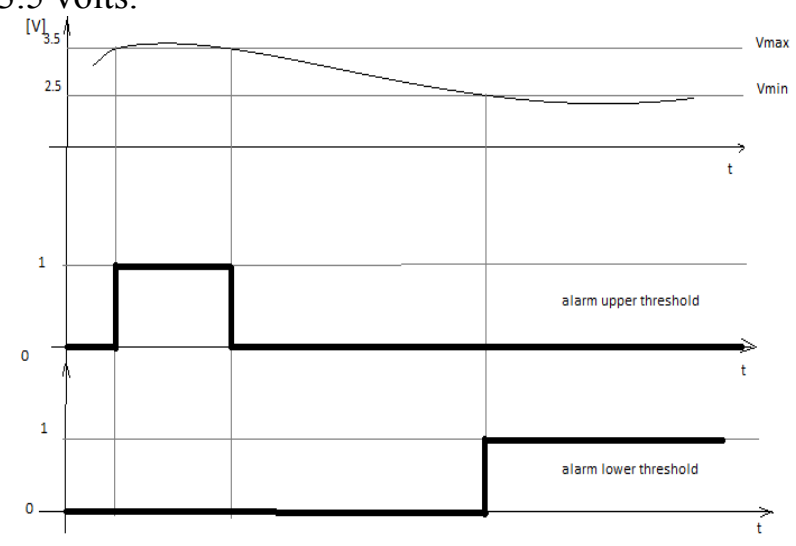

Fig. 17 - Source voltage limit checking.

So, as you can see from the figure above, we have established an upper and a lower threshold in order to have an online fault detection system. One of the data constantly sent by every node is its voltage, so it is relatively easy to predict node malfunction due to power failure related problems.

Another way to deal with the power failure or power fluctuation generated problems is by constantly checking the trend of the measured voltage. If the voltage is climbing or dropping too steeply, it's clear that the node is losing its ability to measure and send data because of exceeding the normal average operating range of the equipment.

\section{CONCLUSIONS}

Using a mobile wireless sensor network for data acquisition inside a building offers many challenges, but also enjoys many benefits from the wireless capability and the mobility of the nodes. This architecture has a very good potential as an alert and monitoring system for a closely monitored environment building as an art museum or even a hospital, a laboratory or a house for homecare monitoring of people with chronic diseases or elderly. Its features are optimized for monitoring over a long period, autonomously, without maintenance and have a good potential for 
upgrading.

One major challenge is how to validate the gathered data from one specific node. The mobility of one or many network nodes comes into play, offering the possibility to place another node in the vicinity of the evaluated node and compare the data to validate them.

Our experiments demonstrated that it is possible to use a few mobile nodes in order to achieve greater coverage and redundancy inside a multi-hop network composed mainly by fixed nodes.

One of the challenges we have to overcome is how to move the sensor nodes relatively to each other in order to send data over a long distance, avoiding obstacles and obtaining a good dropped/received data ratio. We also want to improve our network redundancy by teaching the mobile nodes to take the place of the broken nodes in case of failure.

The future project will also benefit from the GPS capability of the more advanced MTS420, giving us the possibility to operate not only indoor, but also in the surroundings of the monitored building. This step will bring more challenges to the project.

\section{REFERENCES}

[1] M. Ilyas, I. Mahgoub, Eds., Handbook of Sensor Networks. Compact Wireless and Wired Sensing Systems, CRC Press LLC, 2005.

[2] R. Dobrescu, D. Popescu, and M. Nicolae, Mobile node implementation for WSN applications, International Journal of Circuits, Systems and Signal Processing, (2) 1 (2008), pp. 121-130.

[3] M. Al Ameen, R. Islam, and K. Kwak, Energy saving mechanisms for MAC protocols in wireless sensor networks, International Journal of Distributed Sensor Networks, Volume 2010 (2010), Article ID 163413.

[4] S. Song, K. Bok, Y. S. Kwak, B. Goo, Y. Kwak and D. Ko, Dynamic load balancing data centric storage for wireless sensor networks, Sensors, (10) 11 (2010), pp.10329-10338.

[5] D. Kominami, M. Sugano, M. Murata, and T. Hatauchi, Energy-efficient receiver-driven wireless mesh sensor networks, Sensors, (11) 1 (2011), pp.111-137.

[6] D. Popescu, R. Dobrescu, C. Vasilescu, and M. Nicolae, Acquisition, processing and communication module of a WSN-based system for environment monitoring, U.P.B. Scientific Bulletin Series C, 72 (2) 2 (2010). pp. 27-36.

[7] L. Klingbeil, T. Wark, A wireless sensor network for real-time indoor localisation and motion monitoring, Proceedings of the "7th
International Conference on Information Processing in Sensor Networks (IPSN'08)", St. Louis Missouri USA April 22-24 2008, pp. 3950.

[8] www.aal-europe.eu, Ambient Assisted Living Joint Programme (AAL JP), 2008-2013.

[9] R. Isermann, Fault-Diagnosis Systems-An Introduction from Fault Detection to Fault Tolerance, Springer 2005, pp 95-110.

[10] Y. Zeng, C. Sreenan, L. Sitanayah, N. Xiong, J. Hyuk Park, and G. Zheng, An emergencyadaptive routing scheme for wireless sensor networks for building fire hazard monitoring, Sensors, (11) 3 (2011), pp. 2899-2919.

[11] F. Cattivelli, C. Lopes, and A. Sayed, Diffusion Recursive Least-Squares for Distributed Estimation Over Adaptive Networks, IEEE Transactions on Signal Processing, (56) 5 (2008), pp. 1865-1877.

[12] M. I. Struțu, D. Popescu, Wireless sensor network for monitoring applications, Proceedings of the " $\sigma^{\text {th }}$ IEEE International Conference on Intelligent Data Acquisition and Advanced Computing Systems: Technology and Applications, IDAACS 2011", Prague, Czech Republic (September 15-17 2011), pp. 867871.

[13] R. Dobrescu, D. Popescu, M. Dobrescu and M. Nicolae, Integration of WSN-based platform in a homecare monitoring system, Proceedings of "Latest Trends on Communications and Information Technology, 4th International Conference on Communications \& Information Technology (CIT'10)'. Corfu Island, Greece (July 22-25 2010), pp.165-170.

[14] S. Cui, A. J. Goldsmith and A. Bahai, Energyefficiency of MIMO and cooperative MIMO techniques in sensor networks, IEEE Journal on Selected Areas in Communications (22) 6 (2004), pp. 1089-1098.

[15] H. J. Rad, B. Abolhassani, M. Abdizadeh, Lifetime optimization via network sectoring in cooperative wireless sensor networks, Wireless Sensor Network, (2) 12 (2010), pp. 905-909.

[16] MTS420/400 Datasheet, www.memsic.com.

[17] Crossbow, MTS/MDA Sensor Board Users Manual, Revision A, June 2007.

[18] Crossbow, Xmesh User's Manual, Revision C, March 2007.

[19] R. Shorey, A. Ananda, M. C. Chan, and W. T. Ooi, Mobile, Wireless, and Sensor Networks - Technology, Applications, and Future Directions, Wiley-IEEE Press, 2006. 


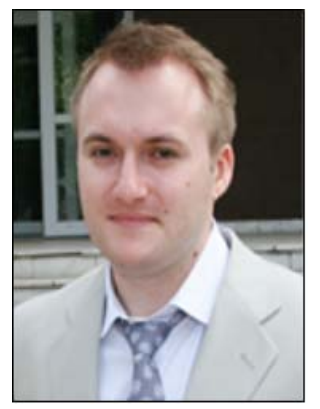

Mircea lonel Strutu was born in 1984 . He received the B.S. and M.S. degrees in control engineering and applied informatics from "Politehnica" University of Bucharest, Bucharest, Romania, in 2008 and 2010, where he is currently working toward the Ph.D. degree in control engineering, under the supervision of Professor Dan Popescu. His main research interests focus on sensor networks and signal processing.
Dan Popescu was born in 1950. He received the M.S. from "Politehnica" University of Bucharest, Bucharest, Romania, in 1974, the M.S. in mathematics from University Bucharest, Bucharest, in 1979, and the Ph.D. degree from "Politehnica" University of Bucharest, in 1986. He is currently Professor with the Control Engineering and Computer Science, "Politenhica" University of Bucharest. His research interests include wireless sensor networks, image processing, modeling and control of systems. He is IEEE member, author or coauthor of 15 books and over 100 scientific papers. 